\title{
Ultrasonography study: Supraspinatus muscle thickness evaluation on dominant versus non dominant shoulder on tennis players
}

\author{
Maria Papandreou ${ }^{1 *}$, Evdokia Billis ${ }^{2}$, George Georgoudis ${ }^{3}$, George Papathanasiou ${ }^{4}$, John Gliatis ${ }^{5}$ \\ ${ }^{1}$ Department of Physiotherapy, Assistant Professor, Physiothereapist, Technological Education Institute of Athens (TEI), Greece \\ ${ }^{2}$ Department of Physiotherapy, Assistant Professor, Physiothereapist, TEI of Western Greece \\ ${ }^{3}$ Department of Physiotherapy, Associate Professor, Physiothereapist TEI of Athens \\ ${ }^{4}$ Department of Physiotherapy, Associate Professor, Physiothereapist, TEI of Athens \\ ${ }^{5}$ Universtity Hospital of Patras, Assistant Professor, MD
}

Received: February 02, 2016; Accepted: June 07, 2016; Published: June 09, 2016

*Corresponding author: Maria Papandreou, PhD, Assistant Professor, Physiotherapy Department, 24 Mitrodorou St, 10446, Athens, Greece, Tel :+306974601171; Fax: +302105385674; E-mail address: mpapand@teiath.gr

\begin{abstract}
The supraspinatus is the most commonly damaged structure in rotator cuff muscles in overhead sports. Supraspinatus injuries affect its strength causing atrophy and reduce shoulder functionality. Ultrasonography imaging (US) provides information on the supraspinatus morphological features, such as muscle thickness (MT). The ability to perform assessment of supraspinatus MT on dominant and non dominant shoulders on tennis players -as overhead athletes- has not been studied and may be of value in assessment and treatment. The purpose of this cross sectional study was to evaluate the differences in the supraspinatus MT between the dominant and non-dominant shoulder on tennis players using brightness-mode US.
\end{abstract}

US images from the supraspinatus MT were recorded in 20 young asymptomatic adult tennis players playing competition leve tennis. Standardized methods in the literature were used to gather US measures on both shoulders. Mean differences were examined using t-test for paired samples

The supraspinatus MT of the dominant shoulder was $2.11 \pm 0.53 \mathrm{~cm}$ and that of the non-dominant $1.38 \pm 0.30 \mathrm{~cm}$. Significant statistical differences were observed on the dominant shoulder compared with the non-dominant one $(t=5.070$, [2-tailed $] p<0.05)$.

The current findings indicate that supraspinatus MT differences using US could provide quantitative measures in tennis players' dominant and non-dominant shoulders.

So, US evaluation may be considered as an outcome measure for assessing supraspinatus atrophy or hypertrophy in rehabilitation and training regimes.

Keywords: Supraspinatus; Ultrasonography; Muscle Thickness; Dominant Shoulder; Non-Dominant Shoulder; Tennis Athletes

\section{Abbreviations}

US=ultrasonography, MT= muscle thickness, b-mode= brightness mode, $\mathrm{CSA}=$ cross sectional area, MRI= magnetic resonance imaging

\section{Introduction}

The supraspinatus, as one of the most important rotator cuff muscles, has been shown to be a substantial dynamic stabilizer of the glenohumeral joint in multiple shoulder positions and activities [1-3]. Supraspinatus muscle injuries are frequently related to overloading or repeated movements in many overhead sports activities such as tennis, squash, volleyball, pitchers [3-5]. It is believed that tennis is one of the overhead sports that expose the dominant shoulder to different kinds of injuries with several symptoms, such as supraspinatus muscle degeneration, tendon tear and calcification [6-8]. Asymptomatic non-elite junior tennis players reported $15 \%$ of minor shoulder abnormalities on dominant shoulder [6,9]. In veterans tennis players supraspinatus injuries have been observed on the dominant shoulder and were found to be related to ageing $[10,11]$. In wheelchair tennis players supraspinatus degeneration has mainly been identified as a high risk factor for further injuries on the dominant shoulder [12]. Supraspinatus muscle and tendon quality is thought to predict the biomechanical strength of the rotator cuff repair and appears to have a major role in determining clinical outcomes [13].

Supraspinatus muscle strength strongly depends on the morphologic properties of the muscle $[14,15]$. One factor that influences the muscle morphological components is the muscle thickness (MT) $[16,17]$. In the literature, few studies have been investigated that the MT is highly related with the cross sectional area (CSA), which is often measured to evaluate muscle atrophy or exercise induced hypertophy after injury and rehabilitation specific regimes [16-18].

The gold standard for measuring the morphological features of supraspinatus is magnetic resonance imaging (MRI) [19]. However, ultrasonography (US) is much less expensive, more readily available and it has been reported to have a good intra 
and inter-tester reliability (greater than 0.80 ) in evaluating the supraspinatus CSA and MT $[16,19,20]$. Tae et al. [20] measured the supraspinatus MT in hemiplegics' patient shoulders by MRI and US and found that US would be helpful in evaluating the strength of the affected upper extremity differences. Temes et al. [17] determined the validity and reliability of measures obtained by US for assessing supraspinatus MT at rest and contracted- in abduction- conditions. Their findings suggest that US may provide an appropriate quantitative measure for changes in the MT of supraspinatus for determining improvements or deterioration in muscle function.

Thus, it is reasonable to assume that US could well be used as an outcome measure for supraspinatus MT changes in physical therapy treatment and functional rehabilitation decisions in overhead athletes for dominant versus non-dominant shoulder comparisons. However, it is important to acknowledge that no studies have been conducted to support this bilateral comparison (between dominant and non-dominant shoulders) of the supraspinatus MT among tennis players as overhead athletes.

Given the above, the purpose of this current study was to determine the differences of the supraspinatus MT between the dominant and non-dominant shoulders on tennis players using brightness-mode (b-mode) US. We hypothesized that the assessment of the supraspinatus MT will provide detectible differences on the dominant versus non-dominant shoulder using US on asymptomatic tennis players. The evaluation of the supraspinatus MT using US could consider as an outcome measure easily monitoring the supraspinatus muscle function.

\section{Methods}

\section{Participants}

Twenty tennis players playing competition level tennis, (12 males and 8 females) were recruited in this study (age: $23.5 \pm 4.1$ years, height: $169.6 \pm 10.4 \mathrm{~cm}$ and weight: $64.7 \pm 10.4 \mathrm{~kg}$ ). In order to be considered eligible to participate in this study, they had to be involved in tennis for more than five years, at a rate of three times per week, with a minimum playing time of one and half hours and they were in regular training season. Exclusion criteria were prior or current history of shoulder injury or pathology and presence of any medical condition of the glenohumeral joint such as infection or systematic disease and trauma. General exclusion criteria according to US scanning were pregnancy, breast feeding and metal parts in the scan area. Subjects were recruited from the sports center of TEI of Athens (TEI-A). The participants who met the inclusion criteria signed an informed consent form which was approved by the local educational institution (TEI-A) ethics committee.

\section{Procedure}

A licensed othropaedic doctor performed a screening of upper extremities to rule out musculoskeletal or other medical problems and identified upper limb dominance.

Corresponding to the side used for playing tennis, 14 of the subjects were right upper extremity dominant and 6 were left upper extremity dominant. All measurements started randomly either from the dominant side or the non-dominant one and both shoulders were evaluated at the same position by the same examiner on the same session. Participants were told not to be engaged in any training activities for at least 24 hours prior to the measures in order to avoid any metabolic responses occurring in supraspinatus muscle.

\section{Examiner}

An experienced physiotherapist- the examiner- on the musculoskeletal shoulder region conducted the measurement procedure. The examiner underwent at least 20 hours of US training on the shoulder region prior to this study.

\section{Experimental design}

All images were acquired with US machine (My Lab60, Esaote, Genova, Italy) and $10 \mathrm{~cm}$, linear probe used in b-mode at 7.5-10MHz.

Participants were seated in a normal dimensions' chair by extending with their shoulders in a neutral position and their elbows in full extension hanging by the side of the body. Image acquisition was performed 3 times on each shoulder and the mean of these was taken for statistical analysis.

In this study, the assessing methods for supraspinatus MT were based on previous standardized protocols (as mentioned below) $[20,21]$.

To standardize the positioning of the US probe, external bone landmarks were used. The procedure started by firstly measuring the anatomical CSA in order to be used a standardized protocol [21] for the assessment of the supraspinatus muscle fascial borders, and secondly the MT.

Firstly, the CSA was measured in the following order (Figure 1). 1. Palpation of the outer and inner edges of the spine of scapular (SS) and drawing a line on the surface along it. 2. Demarcation of the anatomical landmark of the coracoid

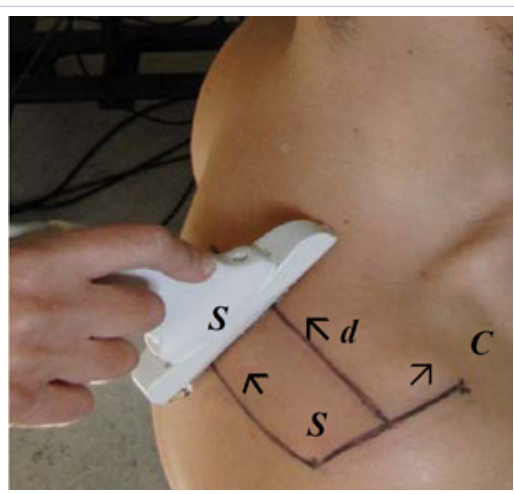

Figure 1: Placement of the US probe for the assessment of the supraspinatus muscle anatomical CSA according to the methodological protocol of Papatzika et al. 2015 and Tae et al. 2012. The probe was placed parallel to CS line and perpendicular to d line to capture the supraspinatus muscle CSA.

C: coracoid process, SS: scapular spine line, CS: line from the coracoid to outer edge of SS, d: parallel line to SS 
process $(\mathrm{C})$ and drawing a line which connected the anatomical landmarks from the coracoid to the outer edge of SS (CS). 3. The probe located parallel to the line CS and the fascial borders of supraspinatus muscle and the scapular notch (SN) was visualized on US monitor and at this point was marked the lateral side of the probe. 4. The distance from the middle landmark of the line CS to the lateral side of probe calculated and drew a parallel line (d) to SS. The probe was placed parallel to CS line and perpendicular to $\mathrm{d}$ line to capture the supraspinatus muscle anatomical CSA in each trial (Figure 1).

Secondly, the measurement of the supraspinatus MT based on the supraspinatus anatomical CSA and was performed manually using the Image J software program by the same observer in the following order. 1. The acoustic shadow of the clavicle was considered as an anterior border of the supraspinatus muscle drawing the vertical line (A). 2 . The scapular spine was considered as a posterior border drawing the vertical line (B) and obtained the midpoint (C) of the distance AB. 3. The superior (D) and inferior (E) most portions of the fascial borders of the muscle considered as the supraspinatus landmarks (superior- inferior) respectively. 4. The vertical distance (DE) from the midpoint (C) corresponded with supraspinatus MT in this study (Figures 2A, 2B). All measurements run smoothly without encountering any problems with any subject.

\section{Statistical analysis}

Effect size along with inferential statistics was assessed between dominant and non-dominant shoulders and based on differences between means. The reliability of the protocol measurements was determined using the interclass coefficientsbased on Pearson Product Moment correlation coefficient (PPM) on 2 testing occasions whichever shoulder, on the first and second day, and the day after the official measurements were started. $\mathrm{T}$ test for paired samples was used to examine the supraspinatus MT for bilateral differences between dominant and non-dominant shoulders. The level of significance for all statistical procedures was set less than $5 \%(\mathrm{p}<0.05)$. The SPSS 16.0 statistical package was used to analyze the data.

\section{Results}

For t-tests the effect size based on differences between means on dominant and non-dominant shoulders' supraspinatus MT using Cohen $d$ and represented as large effect size.

The PPM showed a strong relationship ( $r=0.94)$ between supraspinatus MT scores.

The average supraspinatus MT mean (M), standard deviation (SD) and the standard error of the mean (SEM) for the dominant shoulder was $\mathrm{M} \pm \mathrm{SD}=2.11 \pm 0.53 \mathrm{~cm}, \mathrm{SEM}=0.11$ and for the nondominant $\mathrm{M} \pm \mathrm{SD}=1.38 \pm 0.30 \mathrm{~cm}, \mathrm{SEM}=0.68$.

The mean difference between the MT on the dominant and non-dominant shoulders was found to be $0.73 \mathrm{~cm}, \mathrm{SD}= \pm 0.64$ and the SEM=0.14. The observed $t$ value was found to be 5.070

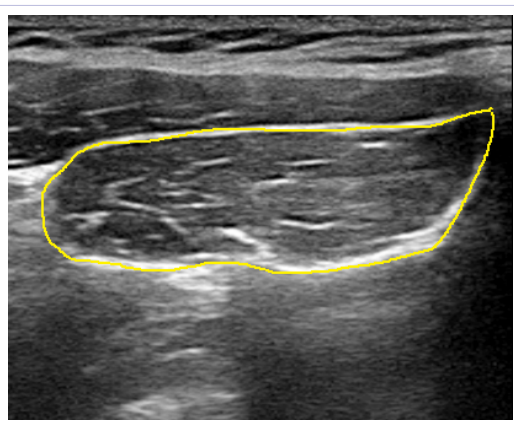

Figure 2a: B- mode US image used for the identifiable area of the supraspinatus muscle.

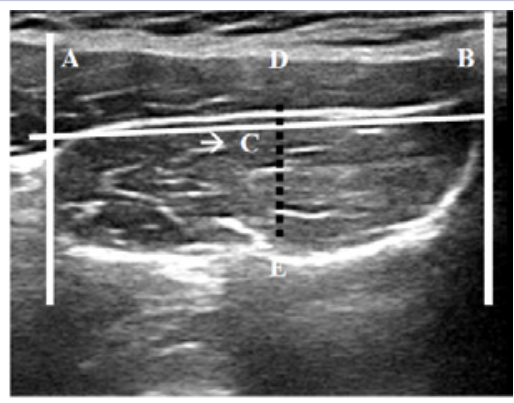

Figure 2b: Methodological protocol (Tae et al. 2012) for the assessment of the supraspinatus MT. DE dotted line considered as the supraspinatus MT in this study.

with an associated probability that approaches .000 , this means statistical significant difference (Significant, $d f=19$, [2-tailed] $\mathrm{p}<0.05$ ) (Table 1).

\section{Discussion}

The objective of this study was to determine the supraspinatus muscle thickness (MT) differences between the dominant and non-dominant shoulders on tennis players using b-mode US. The findings of this study provided significant statistical difference on the supraspinatus MT of the dominant shoulder compared to the non-dominant one $(\mathrm{p}<0.05)$, indicating that the supraspinatus muscle on the dominant shoulder was thicker $(0.73 \mathrm{~cm})$. The coefficient suggested that $94 \%$ of the observed score variance was true score variance. That was, the measurements were consistent across time. Also, the low SEM for supraspinatus MT measurements suggested that US could be used in the evaluation of muscle morphological characteristics.

This difference between the shoulders merely illustrates a diversity pattern or an adaptation of supraspinatus muscle changes on dominant hand in tennis athletes training regimes. Altered patterns of MT have been demonstrated in limited researches based on assessing supraspinatus MT at rest and isometric contracted conditions [17] and on trunk muscles MT of healthy individuals and low back pain patients [22]. The above assumptions may be highly relevant with the assessment or diagnosis of MT supraspinatus morphological alterations in rest, active and pathological conditions. Thus, supraspinatus MT evaluation with US could be considered as an appropriate 
Table 1. Paired samples test. Supraspinatus MT differences between the dominant and the non-dominant shoulders.

\begin{tabular}{|c|c|c|c|c|c|}
\hline \multicolumn{5}{|l|}{ Paired differences } & \multirow{2}{*}{ Significant } \\
\hline Shoulder MT & $\mathrm{M} \pm \mathrm{SD}(\mathrm{cm})$ & SEM & \multicolumn{2}{|c|}{$95 \% \mathrm{CI}$} & \\
\hline Dominant $\mathrm{N}=20$ & $2.11 \pm 0.53$ & 0.11 & Lower & Upper & \multirow{3}{*}{$\begin{array}{l}t=5.070 \\
S(\mathrm{p}<0.05)\end{array}$} \\
\hline Non-Dominant $\mathrm{N}=20$ & $1.38 \pm 0.30$ & 0.68 & \multirow{2}{*}{0.42} & \multirow{2}{*}{1.02} & \\
\hline $\begin{array}{l}\text { MT difference (domi- } \\
\text { nant \& non-dominant) }\end{array}$ & $0.73 \pm 0.64$ & 0.14 & & & \\
\hline
\end{tabular}

$\mathrm{MT}=$ muscle thickness, $\mathrm{M} \pm \mathrm{SD}=$ mean \pm standard deviation, $\mathrm{SEM}=$ standard error of the mean, $\mathrm{CI}=$ confidence interval, $\mathrm{S}=$ significant

quantitative measure for documenting changes in thickness and possibly determining hypertrophy or atrophy to daily or sports activities.

Few studies have been demonstrated that US measurements of the supraspinatus MT are closely related with CSA, which has been correlated with the ability of a muscle to produce force $[16,17,20]$. On the other hand, there are not relevant studies referred on supraspinatus MT changes that have been correlated with muscle strength changes.

Many studies have reported that the supraspinatus is the most commonly affected muscle in rotator cuff pathology on overhead athletes dominant $(\sim 27 \%)$ shoulder versus non-dominant one $(\sim 14 \%)$ one $[5,6,9,23]$. Clinicians traditionally evaluate the imbalances in strength between the dominant versus nondominant, pre and after injured or operated shoulders conditions $[5,7,10,23,24]$. If we use the US as supplementary examination procedure in the clinical evaluation of the supraspinatus we could have more complete quantitative and qualitative information about the muscle condition.

According to the above, our results that MT of the supraspinatus muscle was thicker on the dominant shoulder than the non dominant one should indicate clinically relevant information using US as an outcome measure. US offer the advantages of providing a safe and easy-monitoring measurement of muscle morphological changes in contributing to muscle function and dysfunction conditions.

However, conclusions cannot not be generalised based solely on the present study, as there are no other relevant studies investigating supraspinatus MT on tennis players or other overhead athletes.

Further research should clarify the contribution of the supraspinatus MT changes in active conditions on professional healthy athletes or having shoulder pathologies participating in asymmetrical sports. And also, further research is required to determine the relationship of supraspinatus MT and strength on dominant versus non-dominant shoulder.

Such assessments would allow us to determine morphological changes by US that could be attributable to rehabilitation regimes and training settings.

\section{Conclusion}

The findings of this study provided significant statistical differences on the supraspinatus MT dominant versus the non- dominant shoulder assessing by b-mode US. There are no relevant studies investigating supraspinatus MT differences between the dominant and non-dominant shoulders on overhead athletes; so definite conclusions cannot be made. However, these findings could have implications for clinical researchers using side to side comparisons evaluating the supraspinatus MT changes using US as an outcome measure.

\section{References}

1. Day A, Taylor NF, Green RA. The stabilizing role of the rotator cuff at the shoulder. Responses to external perturbations. Clinical Biomechanics. 2012;27(6):551-556. doi:10.1016/j.clinbiomech.2012. 02.003.

2. Hess AS, Functional stability of the glenohumeral joint. Manual therapy. 2000;5(2):63-71. http://www.ncbi.nlm.nih.gov/pubmed/10903581.

3. Howell SM, Imobersteg AM, Seger DH, Marone PJ. Clarification of the role of the supraspinatus muscle in shoulder function. Journal of Bone and Joint Surgery Am. 1986;68(3):398-404.

4. Kim Y, Bleakney R, Rindlisbacher T, Ravichandiran K, Rosser C, Bounton E. Musculotendinous architecture of pathological supraspinatus: a pilot in vivo ultrasonography study. Clinical Anatomy. 2013;26(2):228235. doi: 10.5535/arm.2011.35.4.524.

5. Trakis EJ, McHugh PM, Caracciolo AP, Busciacco L, Mullaney M, Nicholas JS. Muscle strength and range of motion in adolescent pitchers with throwing-related pain implications for injury prevention. Am J Sports Med. 2008;36(11):2173-8. doi: 10.1177/0363546508319049.

6. Cools MA, Palmans T, Johansson RF. Age-related, sport-specific adaptations of the shoulder girdle in elite adolescent tennis players. Journal of Athletic Training. 2014;49(5): 647-653. doi: 10.4085/10626050-49.3.02.

7. Stanley A, McGann R, Hall J, McKenna L, Briffa NK. Shoulder strength and range of motion in female amateur-league tennis players. Journal of Orthopaedics and Sports Physical Therapy. 2004;34(7):402-409.

8. Yanagisawa O, Okumura K, Torii S. Comparison of the morphology of the rotator cuff muscles across age groups. Clinical Anatomy. 2014;27(3):365-369. doi: 10.1111/j.1600-0838.2012.01477.

9. Tagliafico A, Cadoni A, Bignotti B, Martinoli C. High-resolution ultrasound of rotator cuff and biceps reflection pulley in non-elite junior tennis players: anatomical study. BMC Musculoskeletal Disorders. 2014;15(241):241-247. doi: 10.1186/1471-2474-15-241.

10. Brasseur JL, Lucidarme O, Tardieu M, Tordeur M, Montalvan B, Parier J, et.al. Ultrasonographic rotator cuff changes in veteran tennis players : the effect of hand dominance and comparison with clinical findings. European Radiology. 2004;14(5):857-864. doi: 10.1007/ s00330-003-2116-0.

11. Smith TO, Back T, Toms AP, Hing CB. Diagnostic accuracy of ultrasound for rotator cuff tears in adults: A systematic review and metaanalysis. Clinical Radiology. 2011;66(11):1036-1048. doi: 10.1007/ s00296-011-2229-8.

12. Jeon IH, Kochhar H, Lee JM, Kyung HS, Min WK, Cho HS. Ultrasonographic evaluation of the shoulder in elite wheelchair tennis players. J Sport Rehabil. 2010;19(2):161-172.

13. Strobel K, Hodler J, Meyer D, Pfirrmann C, Pirkl C, Zanetti M. Fatty Atrophy of Supraspinatus and Infraspinatus Muscles: Accuracy of US. Radiology. 2005;237(2):584-589. doi: 10.1148/radiol.2372041612.

14. Lieber RL, Fridén J. Functional and clinical significance of skeletal muscle architecture. Muscle and Nerve. 2000;23(11): 1647-1666. 
15. Tempelhof S, Rupp S, Seil R. Age-related prevalence of rotator cuff tears in asymptomatic shoulders. Journal of Shoulder and Elbow Surgery. 1999;8(4):296-299.

16. Schneebeli A, Egloff M, Giampietro A, Clijsen R, Barbero M. Rehabilitative ultrasound imaging of the supraspinatus muscle: Intra-and interrater reliability of thickness and cross sectional area. Bodywork and Movement Therapies. 2014;18(2):266-272. doi: 10.1016/j.jbmt.2013.09.009.

17. Temes W, Temes Clifton A, Hilton V, Girard L, Strait N, Karduna. Reliability and validity of thickness measurements of the supraspinatus muscle of the shoulder: An ultrasonography study. J Sport Rehabil. 2014;8:1-5. doi.org/10.1123/jsr.2013-0023.

18. Stokes M, Hides J, Nassiri DK. Musculoskeletal ultrasound imaging: diagnostic and treatment aid in rehabilitation. Physical Therapy Review. 1997;2(2):73-92. doi:10.1179/ptr.1997.2.2.73

19. Whittaker JL, Stokes M. Ultrasound imaging and muscle function. Journal of Orthopaedics and Sports Physical Medicine. 2011;41(8):572-280. doi:10.2519/jospt.2011.3682.

20. Tae IY, Han SI, Kim SJ, Jin RJ, Han SJ. Reliability of the supraspinatus muscle thickness measurement by ultrasonography. Annals of Rehabilation and Medicine. 2012;36(4):488-495. doi.org/10.5535/ arm.2012.36.4.488.

21. Papatzika F, Papandreou M, Ekizos A, Panteli C, Arampatzis A. Reliability and limits of agreement of the supraspinatus muscle anatomicalcross-sectional area assessment by ultrasonography. Ultrasound in Medicine and Biology. 2015;41(7):1821-1826. dx.doi. org/10.1016/j.ultrasmedbio.2015.02.017.

22. Teyhen DS ${ }^{1}$, Williamson JN, Carlson NH, Suttles ST,O'Laughlin SJ, Whittaker JL, et,al. Ultrasound characteristics of the deep abdominal muscles during the active straight leg raise test. Archives of Physical Medicine and Rehabilitation. 2009;90(5):761-767. doi: 10.1016/j.apmr.2008.11.011.

23. Kibler WB, Chandler TJ, Shapiro R, Conuel M. Muscle activation in coupled scapulohumeral motions in the high performance tennis serve. Br J Sports Med. 2007;41(11):745-749. doi:10.1136/ bjsm.2007.037333.

24. Chung WS, Kim HS, Tae SK, Yoon PJ, Choi JA, Oh HJ. Is the supraspinatus muscle atrophy truly irreversible after surgical repair of rotator cuff tears? Clinical Orthopaedic and Surgery. 2013;5(1):55-65. doi: 10.4055/cios.2013.5.1.55 